exacerbations requiring oral steroids (OR per genotypic step $1.19 ; \mathrm{p}=0.008$ )

Conclusions Analysis of these four SNPs could enable clinicians to identify patients at higher risk of a severe asthma phenotype, potentially helping tailor strategies for improved asthma control.

"S14- BTS Medical Student Award Winner

\section{S15 MEASURING BRONCHODILATOR RESPONSE BY INTERRUPTER TECHNIQUE TO PREDICT RESPONSE TO INHALED STEROID THERAPY IN WHEEZY PRESCHOOL CHILDREN}

${ }^{1} \mathrm{R}$ Willson, ${ }^{1} \mathrm{C}$ Olden, ${ }^{1} \mathrm{~L}$ Symes, ${ }^{2} \mathrm{~N}$ Beydon, ${ }^{3} \mathrm{E}$ Lombardi, ${ }^{4} \mathrm{D}$ Wertheim, ${ }^{1} \mathrm{P}$ Seddon. ${ }^{1}$ Royal Alexandra Children's Hospital, Brighton, UK; ${ }^{2}$ Hôpital Armand Trousseau, Paris, France; ${ }^{3}$ Ospedale Pediatrico "Anna Meyer", Florence, Italy; ${ }^{4}$ Kingston University, Kingston, UK

\subsection{6/thoraxjn-2015-207770.21}

Managing recurrent wheezing in preschool children is problematic, with conflicting evidence on the effectiveness of asthma therapy. One reason is the heterogeneous nature of preschool wheeze: distinguishing "transient viral wheeze" from asthma at presentation is currently impossible. Bronchodilator response (BDR) measured by interrupter resistance (Rint) is greater in preschool children with diagnosed asthma than in healthy controls, but the usefulness of Rint in clinical practice has not been studied. We aimed to assess whether measuring BDR using Rint can predict clinical response to inhaled corticosteroids (ICS).

We studied children aged 2 years to 1 . Response to ICS was defined as reduction in daily symptom score of at least $0.26 .^{2}$ Association between BDR and ICS response was assessed using chi-square test. We also assessed the relationship between measured BDR and the caregiver's assessment.

121 children were recruited, 59 completed the full study (27 withdrew, 10 were unable to perform Rint, 18 failed to meet $4 \mathrm{w}$ symptom threshold, 7 commenced ICS or Montelukast by clinician before $4 \mathrm{w}$ ). Positive BDR at baseline was demonstrated by 33 children, and was significantly associated with a clinical response to ICS $(p<0.05)$. There was no relationship between objective BDR and caregiver's rating of bronchodilator efficacy.

Our findings suggest that measuring BDR using Rint may help in making pragmatic decisions on commencing asthma preventer therapy in wheezy preschool children. Further work is needed to assess whether Rint BDR measurement may have the potential to distinguish those with transient wheeze from those with asthma.

\section{REFERENCES}

1 Pediatr. Pulmonol. 2010;45:633-638

2 Pediatrics 2001;108:E48

\section{S16 OUTCOMES OF MULTIPLE TRIGGER WHEEZE AND EXCLUSIVE VIRAL WHEEZE IN EARLY CHILDHOOD: A COMPARISON ACROSS TWO POPULATION COHORTS}

${ }^{1} \mathrm{CN}$ Cochrane, ${ }^{2} \mathrm{BD}$ Spycher, ${ }^{3} \mathrm{R}$ Granell, ${ }^{3} \mathrm{JAC}$ Sterne, ${ }^{4} \mathrm{M}$ Silverman, ${ }^{2} \mathrm{AM}$ Pescatore, ${ }^{2}$ EA Gaillard, ${ }^{2}$ CE Kuehni, ${ }^{3} \mathrm{~J}$ Henderson. ${ }^{1}$ Royal United Hospital, Bath, UK; ${ }^{2}$ Institute of Social and Preventive Medicine (ISPM), University of Bern, Bern, Switzerland; ${ }^{3}$ School of Social and Community Medicine, University of Bristol, Bristol, UK; ${ }^{4}$ Division of Child Health, Department of Infection, Immunity and Inflammation, University of Leicester, Leicester, UK

10.1136/thoraxjnl-2015-207770.22
Introduction Early childhood wheeze has been classified according to triggers; exclusive viral wheeze (EVW) and multiple trigger wheeze (MTW). It has been proposed that these phenotypes differ in their aetiology, severity and prognosis.

Objectives To examine the prevalence and symptom severity of EVW and MTW in 2-6 year olds. To evaluate the stability of these phenotypes over time, and their association with later wheeze.

Method In two longitudinal birth cohorts comprising 18,362 individuals, data on respiratory symptoms were collected at age 2, 4 and 6 years. Parent-reported triggers were used to classify wheeze as EVW or MTW for each 2-year period. Logistic regression analysis was used to estimate odds ratios for current wheeze and relative risk ratios for wheeze phenotypes versus no wheeze, at follow-up compared with baseline.

Results At 2 years $17.6 \%$ and 22.6\% (cohorts 1 and 2 respectively) had wheeze, of which $55.2 \%$ and $56.3 \%$ had MTW. At 6 years $69.7 \%$ and $75.7 \%$ of children with wheeze had MTW.

Among children with wheeze at baseline, 58-76\% with EVW and $46-67 \%$ with MTW were in remission 2 years later (cohort 1) and $14-20 \%$ and $4-11 \%$ (cohort 2).

MTW had greater reported symptom-severity at all timepoints compared with EVW.

When adjusted for symptom-severity, children with EVW at baseline had relative risk ratios (RRR) of 2.9-7.4 and 4.1-15.5 (cohorts 1 and 2 respectively) for EVW and RRR 1.7-2.9 and 1.6-4.0 for MTW at follow-up. Children with MTW at baseline had RRR of 3.1-6.2 in cohort 1 and 3.6-15.6 in cohort 2 for MTW and 1.1-2.7 and 1.4-7.0 respectively for EVW at followup.

Conclusions When adjusted for symptom severity, wheezing phenotypes based on reported triggers remained stable between 2-6 years of age. Symptom-severity may be a more important determinant than triggers of future wheeze classification in young children.

\section{S17 DO PRE-SCHOOL LUNG VENTILATION SCANS PREDICT OUTCOME BY 6 YEARS OF AGE IN CHILDREN WITH CYSTIC FIBROSIS (CF)?}

${ }^{1} \mathrm{R}$ Yahia, ${ }^{2} \mathrm{~L}$ Viviani, ${ }^{2} \mathrm{~S}$ Carr, ${ }^{3} \mathrm{~A}$ Bush. ${ }^{1}$ St. George's University of London Medical Programme Delivered by the University of Nicosia, Nicosia, Cyprus; ${ }^{2}$ Royal Brompton Hospital, London, UK; ${ }^{3}$ Imperial College London, National Heart and Lung Institute, Royal Brompton Hospital, London, UK

\subsection{6/thoraxjn-2015-207770.23}

Introduction and objectives Progressive respiratory disease accounts for most of the mortality and morbidity in CF. Identification of early lung disease is imperative to recognise young patients who are at high risk of developing future lung damage. The London CF collaboration has shown that infant pulmonary function at one and at two years is essentially normal, and one year HRCT has mild abnormalities only, so new markers need to be identified. We have used ventilation scans (VS) at the CF annual assessment in infants too young to perform standard pulmonary function tests; VS are more sensitive than chest radiography, and have been used to guide immediate management. We hypothesised that an abnormal pre-school lung VS predicted worse spirometry by age six years in CF children.

Methods Data from children born after 2000 under the care of the RBH were retrieved from hospital databases and Port CF. We recorded demographics (gender, age, CFTR genotype, 
weight, height, ethnicity) and spirometry nearest to 6 years of age because repeatable measurements in a clinical context are feasible at this age. The primary outcome was $\mathrm{FEV}_{1} \%$ predicted (\%p) (GLI reference equation (http://www.lungfunction.org/); and VS at annual assessment. Between 1-5 scans were performed prior to the age 6 year spirometry, and were independently reported as normal or abnormal (at least one abnormal VS). Statistical analysis was performed using Student t test. $\mathrm{P}<0.05$ was considered significant.

Results 143/217 children (72 females, 71 males) had data on VS and spirometry available; mean age at first spirometry was 6.36 (range 5.0-7.6). The remaining 73 were excluded due to late diagnosis, moving away before the first reliable spirometry, or first being seen later than the window for ventilation scans $(1-5$ years). Children with $\geq 1$ abnormal VS had a statistically significant reduction in lung function (mean FEV1\% p 83.4\%) when compared with children with normal ventilation scans (mean FEV1\% p\%89.6), $\mathrm{P}=0.03$ (Figure 1).

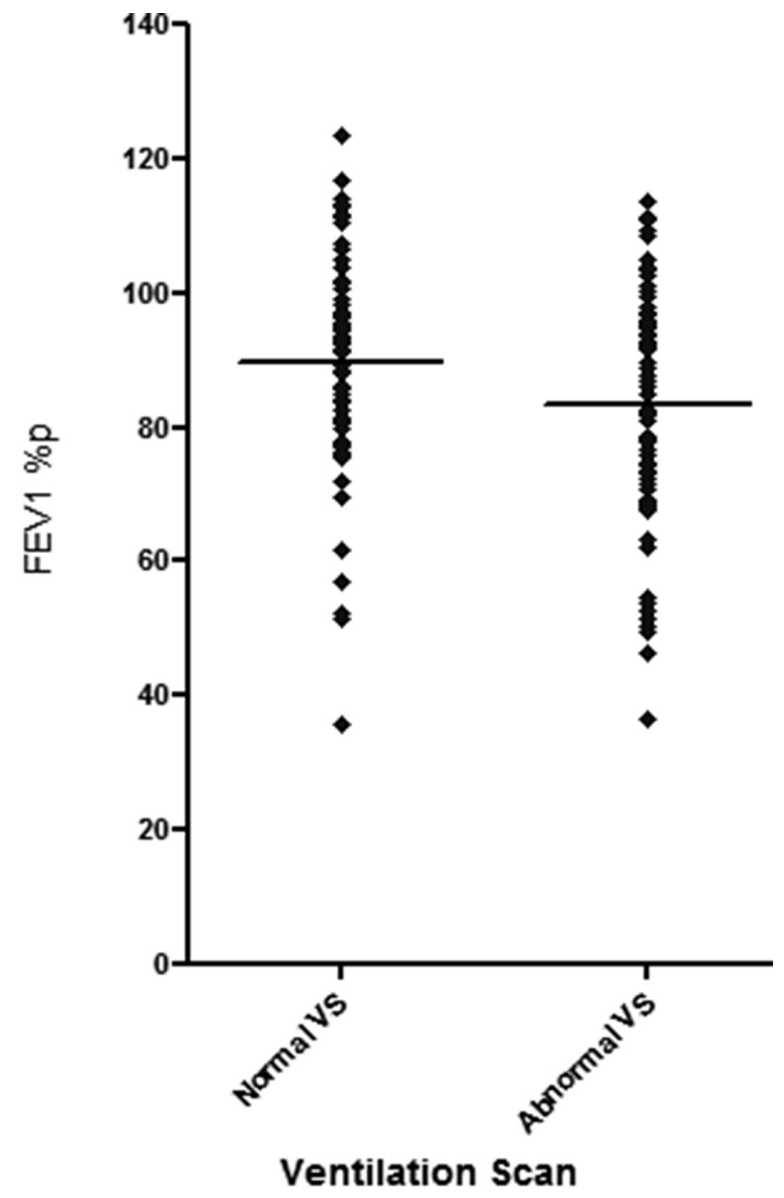

Abstract S17 Figure 1 Dot plot showing\%p comparison between those with normal and abnormal scans. The black horizontal lines are the group means

Conclusion Although abnormal VS predict abnormal first spirometry, the overlap between the two groups means that VS are not a useful clinical tool to delineate a high risk group.

\section{S18 IVACAFTOR TREATMENT IN PRESCHOOL CHILDREN WITH CYSTIC FIBROSIS AND A CFTR GATING MUTATION: EXTENDED EVALUATION}

${ }^{1} \mathrm{JC}$ Davies, ${ }^{2} \mathrm{~S}$ Cunningham, ${ }^{3} \mathrm{KW}$ Southern, ${ }^{4} \mathrm{~S}$ Robertson, ${ }^{5} \mathrm{Y}$ Green, ${ }^{5} \mathrm{~J}$ Cooke, ${ }^{5} \mathrm{M}$ Higgins, ${ }^{6} \mathrm{M}$ Rosenfeld. ${ }^{1}$ Imperial College \& Royal Brompton \& Harefield NHS Foundation Trust, London, UK; ${ }^{2}$ Royal Hospital for Sick Children, Edinburgh, UK; ${ }^{3}$ Alder Hey Children's Hospital, Liverpool, UK; ${ }^{4}$ Vertex Pharmaceuticals Incorporated, Boston, USA; ${ }^{5}$ Vertex Pharmaceuticals (Europe) Limited, Milton Park, UK; ${ }^{6}$ Seattle Children's Hospital, Seattle, USA

\subsection{6/thoraxjn-2015-207770.24}

Introduction and objectives Ivacaftor, a cystic fibrosis transmembrane conductance regulator (CFTR) potentiator, has demonstrated improved outcomes in patients aged $\geq 6$ years with cystic fibrosis (CF) and a CFTR gating mutation. The phase 3, openlabel, 24-week KIWI study assessed safety and pharmacokinetics of ivacaftor (50- or $75-\mathrm{mg}$ granules twice daily [bid] for weight. Methods Patients who completed KIWI could enrol in KLIMB (an 84-week, open-label extension study). Dosing was the same as in KIWI for patients aged $<6$ years; for patients aged $\geq 6$ years, dosing was 150-mg tablets bid.

Results KIWI enrolled 34 patients (mean age, 3.2 years); 33 enrolled in KLIMB. Cough was the most common AE in both KIWI (56\%) and KLIMB (64\%). Over the total 72-week treatment period, 8 patients had ALT or AST elevations of $>8 \times$ the upper limit of normal (ULN), 6 of whom had liver function tests (LFTs) $>2 \times$ ULN at pretreatment baseline. Six of the 8 patients with LFTs $>8 \times$ ULN had drug interruption; study drug was subsequently resumed. In total, 3 patients permanently discontinued study drug (elevated LFTs, $\mathrm{n}=2$; needle phobia, $\mathrm{n}=1$ ). Improvements in sweat chloride, faecal elastase-1, and immunoreactive trypsinogen were maintained over 72 weeks. Overall improvements from baseline in other exploratory outcome measures were also observed (Table 1).

Conclusions A favourable overall safety profile was demonstrated with ivacaftor during extended follow-up in preschool patients with CF. Reported adverse events were consistent with the known safety profile of ivacaftor; additional monitoring of liver function may be required in this age group, particularly in patients with a history of elevated LFTs. Improvements in markers of pancreatic function and sweat chloride were sustained over the extended follow-up.

\begin{tabular}{lll}
$\begin{array}{l}\text { Abstract S18 Table 1 } \\
\text { measures }\end{array}$ & Summary of exploratory efficacy outcome \\
\hline $\begin{array}{l}\text { Absolute change from KIWI } \\
\text { baseline, mean (SD) }\end{array}$ & KIWI (Wk 24) & KLIMB (Wk 72) \\
\hline Sweat chloride, mmol/L & $-46.9(26.2) P<0.0001$ & $-45.4(31.3) P<0.0001$ \\
Weight, kg & $1.4(0.6) P<0.0001$ & $3.2(1.1) P<0.0001$ \\
Weight-for-age z-score & $0.2(0.3) P<0.0001$ & $0.1(0.5) P=0.3$ \\
BMI, kg/m ${ }^{2}$ & $0.3(0.5) P=0.002$ & $-0.2(0.7) P=0.2$ \\
BMI-for-age z-score & $0.4(0.4) P<0.0001$ & $0.1(0.5)=0.5$ \\
Stature, cm & $3.3(1.2) P<0.0001$ & $10.2(1.8) P<0.0001$ \\
Stature-for-age z-score & $-0.01(0.3) P=0.8$ & $0.1(0.3) P=0.01$ \\
Fecal elastase-1, $\mu \mathrm{gg} / \mathrm{g}^{\mathrm{a}}$ & $99.8(138.4)$ & $101.9(152.3)$ \\
Immunoreactive trypsinogen, $\mathrm{ng} / \mathrm{mL}^{\mathrm{a}}$ & $-20.7(24.0)$ & $-18.9(28.0)$
\end{tabular}

$P$ values for both KIWI and KLIMB assessed for absolute change from KIWI baseline. ${ }^{a}$ Significance was not assessed for these changes. 